\title{
Endothelial cell DNA transfer and expression using Petri dish electroporation and the nonreplicating vaccinia virus/T7 RNA polymerase hybrid system
}

\author{
EW Lewis ${ }^{1}$, TJ Rudo ${ }^{1}$, MA Rahman St John², JL Chu' ${ }^{1}$, AW Heinze ${ }^{1}$, BH Howard ${ }^{2}$ and KA Engleka ${ }^{1}$ \\ ${ }^{1}$ Department of Physiology, Jefferson Medical College, Thomas Jefferson University, Philadelphia, PA: and ${ }^{2}$ Laboratory of Molecular \\ Growth Regulation National Institute of Child Health and Human Development, National Institutes of Health, Bethesda, MD, USA
}

The nonreplicating vaccinia virus MVA/T7 RNA polymerase hybrid system was tested with Petri dish electroporation for ectopic gene expression in human umbilical vein endothelial cells (HUVECs). A range of voltages (150$450 \mathrm{~V})$, pulse times (10-40 ms), DNA concentrations (0$20 \mu \mathrm{g} / \mathrm{ml})$ and infection levels (0-15 multiplicities of infection) were tested for effects on $T 7$ promoter-directed chloramphenicol acetyltransferase (CAT) activity after MVA/T7RP infection. MVA/T7RP-directed expression was transient and at least 10000 -fold in excess of nonviral, cytomegalovirus enhancer-directed expression. Use of a Petri dish electrode with the MVA/T7RP system showed increased viability compared with a cuvette electrode. Overexpression of interleukin-2 alpha subunit (IL2R $\alpha$ ) pro- tein followed by anti-IL2R $\alpha$-directed magnetic immunoaffinity cell sorting allowed isolation of the transfected population. The high fidelity of cellular sorting was shown by segregation of CAT activity in the IL2R $\alpha$-sorted population after transfection of $T 7$ promoter-directed bicistronic IL2R $\alpha / C A T$ DNA. Expression of a panel of proteins including the fluorophore green fluorescent protein as detected by fluorescence microscopy and p21 $1^{c i p 1}, p 27^{k i p 1}, p p 60^{c-s r c}$ FGF-1, pRb, $p 107$ and pRb2/p130 proteins was also achieved. Thus, use of the nonreplicating vaccinia virus/T7 RNA polymerase expression system with Petri dish electroporation is feasible for certain applications for the manipulation of HUVECs by gene transfer.

Keywords: endothelial cell; vaccinia virus; electroporation; transfection; expression

\section{Introduction}

Cultured primary human umbilical vein endothelial cells (HUVECs) are attractive for the study of endothelial cell function in vitro because they: (1) are readily available and well characterized relative to endothelial cells of other human tissue origin; and (2) display growth factor and extracellular matrix-mediated phenotypes reflective of normal human endothelial cell growth and morphogenic pathways in vivo. However, HUVECs are resistant to conventional gene transfer techniques and selection methods relative to immortalized cell lines and nonhuman endothelial cells. ${ }^{1-5}$ Cultured HUVECs exhibit decreased transfection efficiencies, an intolerance to transfection and selection conditions and low expression levels after genes are delivered. The inefficiency of HUVEC transgene expression using nonviral, DNA transfection is illustrated by expression levels $1 \%$ relative to those in a commonly used human immortalized cell line and maximized transfection efficiencies reported as low as $0.68-2 \% .3,6$ These barriers have limited the manipulation of HUVECs by the overexpression of both wild-type and mutant proteins or the application of 'knock-out/down' strategies using gene transfer. This

Correspondence: KA Engleka, Department of Physiology, Jefferson Medical College, Thomas Jefferson University, 1020 Locust Street, Philadelphia, PA 19107-6799, USA

Received 5 November 1998; accepted 1 May 1999 has hampered the study of endothelial cell function in vitro and the development of gene therapeutic methods in vivo where the endothelium is an attractive target. ${ }^{7}$ Improved approaches of gene delivery and expression are required to allow experimental and therapeutic manipulation of HUVECs and other normal human endothelial cells by ectopic gene overexpression. The use of viral methods as in adenoviral or retrovirus-mediated gene transfer offers attractive alternatives. ${ }^{8-10}$

Another viral method for gene expression in mammalian cells utilizes vaccinia virus (VV). The nonreplicating vaccinia virus/T7 RNA polymerase hybrid expression system results in high expression levels in eukaryotic cells hundreds-of-fold in excess of conventional eukaryotic expression systems. ${ }^{11-14}$ This system utilizes infection with the cytoplasmic, nonreplicating VVmodified vaccinia Ankara engineered to express T7 RNA polymerase (MVA/T7RP). The T7RP expressed from a viral promoter drives transcription of transfected DNA vectors containing T7 promoter (PT7)-directed sequences. ${ }^{15}$ Thus, MVA/T7RP expression is less dependent on eukaryotic components than conventional expression systems and is potentially cytoplasmic in nature, bypassing nuclear barriers to transfection.

As compared with precipitation-based transfection methods, including liposome-mediated DNA transfection, advantages of electroporation include rapidity of use, avoidance of chemical toxicity and applicability in vivo. ${ }^{16}$ However, electroporation methods have yielded 
low transfection efficiencies and limited expression levels in HUVECs. ${ }^{1,3,17}$ Furthermore, cuvette electroporation requires manipulation of cells into a cuvette. This may introduce artifactual effects in addition to the receiving of an electric shock that may inhibit or delay subsequent gene expression and perturb cellular function. In contrast, a Petri dish electrode bypasses the necessary cellular manipulation for cuvette electroporation.

The high expression levels achieved by the MVA/T7RP hybrid system coupled with the benefits of Petri dish electroporation might overcome barriers to ectopic gene expression displayed by HUVECs. The following experiments evaluate the efficacy of such an approach. The result is a gene expression method that retains advantages of electroporation and allows manipulation of HUVECs by gene transfer.

\section{Results}

\section{Optimization of electroporation conditions}

The effects of voltage and pulse times were tested to establish optimized electroporation conditions for HUVECs using a Petri dish electrode. HUVECs were transiently transfected with a DNA plasmid containing the chloramphenicol acetyltransferase (CAT) gene under control of the T7 promoter (PT7/CAT) and infected with recombinant MVA modified to express T7RP (MVA/T7RP). ${ }^{14}$ The electroporation conditions tested were a single square-wave pulse at 150,350 or $450 \mathrm{~V}$ each at 10,20 or $40 \mathrm{~ms}$ (Figure 1 ). Readily detectable CAT activity was observed at $350 \mathrm{~V}$ and highest expression was observed at $450 \mathrm{~V}$. Twenty milliseconds was utilized as a pulse length in all subsequent experiments.

Potential toxic effects of the transfection and infection conditions were assessed by quantification of viable HUVECs as measured by cell cytometry and trypan blue exclusion. For Petri dish electroporation HUVECs either remained untreated or were MVA/T7RP infected alone, electroporated alone at 350 or $450 \mathrm{~V}$, or infected and elec-

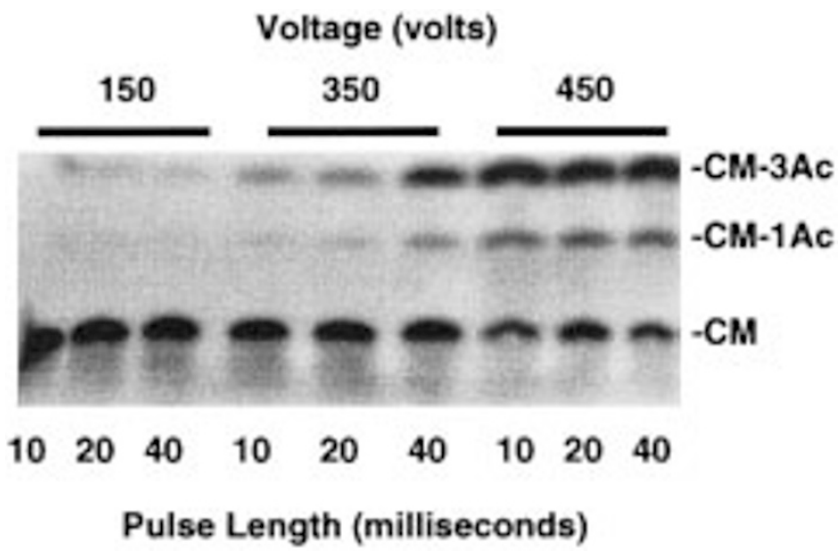

Figure 1 CAT expression as a function of voltage and pulse length HUVECS were electroporated with a single square-wave pulse with $50 \mu \mathrm{g}$ of PT7/CAT DNA in $10 \mathrm{ml}$ using a Petri dish electrode and the conditions shown. The cells were then infected with 10 MOI of MVA/T7RP and harvested $24 \mathrm{~h}$ after transfection. Gene expression was measured in HUVEC extracts by radioactive CAT assay. The unmodified $\left[{ }^{14} \mathrm{C}\right]$ chloramphenicol $(C M)$ was separated from $\left[{ }^{14} \mathrm{C}\right]$ chloramphenicol 1-monoacetate $(C M-1 A c)$ and $\left[{ }^{14} \mathrm{C}\right]$ chloramphenicol 3-monoacetate $(C M-3 A c)$ by thin-layer chromatography and the resultant autoradiograph is shown. troporated at 350 or $450 \mathrm{~V}$ (Figure 2). Results were compared with samples transfected by cuvette electroporation using optimized conditions. The cuvette electroporation conditions included removal of the cells from cultureware and placement into a 4-mm gap cuvette without or with a subsequent $180 \mathrm{~V}$ square-wave pulse for $20 \mathrm{~ms}$ using $5 \mu \mathrm{g}$ of PT7/CAT DNA in $800 \mu \mathrm{l}$ electroporation buffer. These conditions are similar to those previously published as optimal for HUVECs using cuvette electroporation. ${ }^{17}$ As typified by results shown in Figure 2a, both Petri dish and cuvette electroporation methods resulted in less viable cells compared with an untreated control. However, the Petri dish electrode showed $23 \%$ more viable cells relative to the cuvette electrode using the applied transfection and infection conditions (Figure 2a). For both electrodes, viable cell number was less after concomitant transfection and infection compared with electroporation or infection alone.

The results were compared with those achieved in samples using nonviral expression of CAT DNA under control of the powerful cytomegalovirus (CMV) enhancer after Petri dish or cuvette electroporation. Nonviral CMV-directed expression also displayed a decrease in viable cells relative to untreated control that, unlike the MVA/T7RP system, was similar regardless of the electrode type (Figure 2b).

The samples that produced the viability data displayed in Figure 2 were further tested for CAT expression. Similar amounts of MVA/T7RP-mediated expression were observed for Petri dish and cuvette electroporation using normalized amounts of protein (Figure 3). However, total protein recovery for the cuvette-electroporated sample was approximately one-half that of the Petri dish electrode (data not shown) consistent with the decreased number of viable cells displayed by the cuvette-electroporated sample (Figure 2). This is indicative of a decreased amount of total transgene expression after cuvette electroporation. Accordingly, quantification of CAT activity by imaging analysis showed a $48 \%$ increase in total CAT activity with the use of the Petri dish electrode relative to the cuvette electrode (data not shown).

The MVA/T7RP-mediated expression levels were also compared with nonviral expression after transfection of CMV/CAT DNA. Nonviral expression was undetectable under similar assay conditions (5 ng lysate for $30 \mathrm{~min}$ ) regardless of the electrode used (Figure 3). However, the CMV/CAT DNA was fully functional as activity was detected using 10000 -fold more protein $(50 \mu \mathrm{g})$ and a sixfold longer assay time $(3 \mathrm{~h})$. Thus, activity of the nonviral CMV-mediated system was less than $0.0001 \%$ that achieved by the MVA/T7RP system under these conditions.

\section{Effect of MVA/T7RP infection levels}

The expression of transgenes in the nonreplicating MVA/T7RP system is dependent on viral infection. ${ }^{11,14}$ Not only is the virus involved in the delivery and expression of T7RP but other viral factors are required to enhance T7RP expression. ${ }^{11,18}$ After Petri dish electroporation and infection with a range from 1 to 15 multiplicities of infection (MOI) of MVA/T7RP, a linear dosedependent increase in CAT activity was observed in HUVECs (Figure 4a). Standard conditions for future experiments were chosen at $10 \mathrm{MOI}$. This balanced the statistical probability of infecting all cells against unwanted cytopathic effects caused by over-infection. 


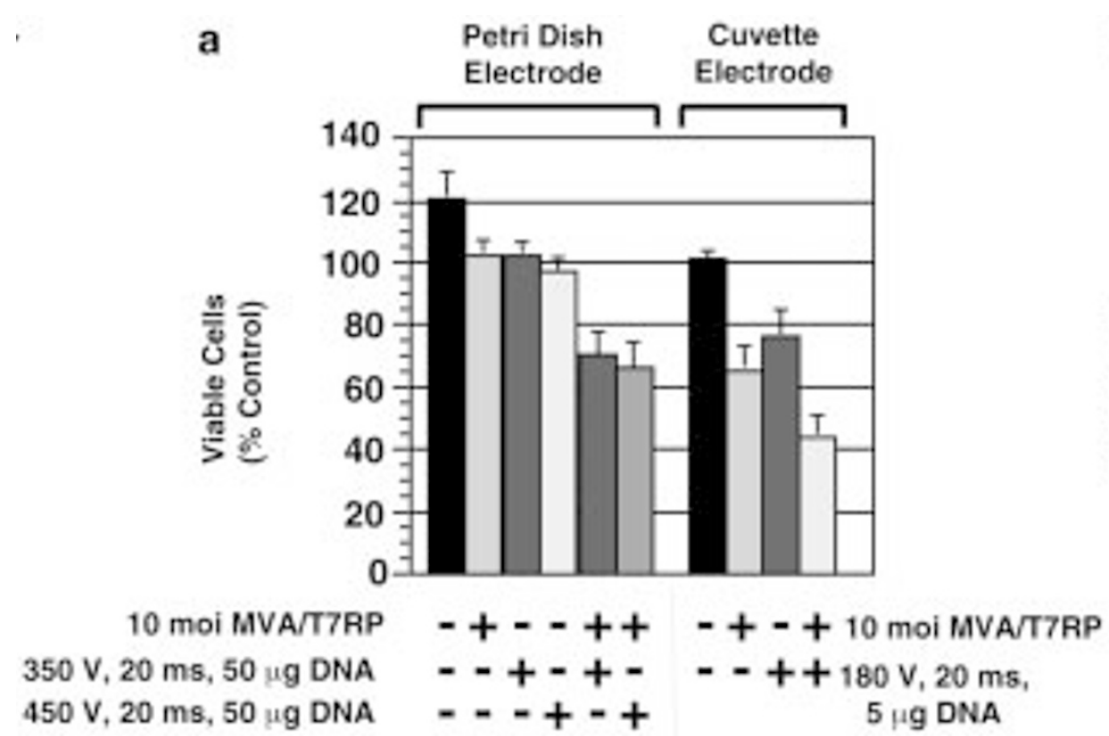

b

Figure 2 Effect of infection and transfection conditions on cell number. (a) HUVECs were either untreated or MVA-T7RP infected alone or together with DNA transection using Petri dish or cuvette electroporation as shown. Attached cells were harvested $16 \mathrm{~h}$ after transfection, stained with trypan blue and viable cells counted in triplicate by cell cytometry. The number of viable cells was compared with that obtained just before infection/transfection and expressed as a percentage as shown. The experiment was repeated three times with two different HUVEC isolates with similar results. (b) Using samples done in parallel with those in (a), HUVECs remained uninfected but were transfected with CMV/CAT DNA using either a Petri dish (50 $\mu g$ DNA in $10 \mathrm{ml}$ ) or cuvette $(5 \mu \mathrm{g} D N A$ in $0.8 \mathrm{ml}$ ) electrode. Cells were harvested and the number of viable cells quantified as in (a). P, Petri dish electrode; C, cuvette electrode.

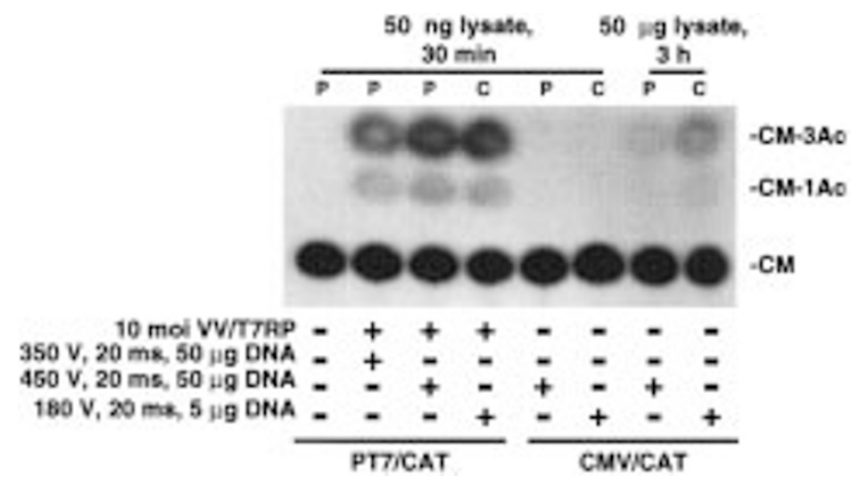

Figure 3 Effect of infection and transfection conditions on CAT expression. Cells used to produce the results depicted in Figure 2 were tested for gene expression using the CAT assay conditions shown. P, Petri dish electrode; C, cuvette electrode; $C M,\left[{ }^{14} \mathrm{C}\right]$ chloramphenicol; $C M-1 A c$, $\left[{ }^{14} \mathrm{C}\right]$ chloramphenicol 1-monoacetate; $(\mathrm{CM}-3 \mathrm{Ac}),\left[{ }^{14} \mathrm{C}\right]$ chloramphenicol 3-monoacetate.

\section{Effect of DNA concentration}

DNA concentrations from 25 to $200 \mu \mathrm{g}$ per $100-\mathrm{mm}$ dish were tested for Petri dish electroporation (Figure 4b). Since a minimum buffer volume of $10 \mathrm{ml}$ was required to immerse the Petri dish electrode completely, this represented $2.5-20 \mu \mathrm{g} / \mathrm{ml}$ of DNA. This is comparable with the range of DNA used in cuvette electroporation in our laboratory. Increasing the amount of DNA electroporated resulted in corresponding increases in CAT expression (Figure 4b).

\section{Kinetics of expression}

The replication-deficient MVA/T7RP system displays transient expression for 4-5 days. $^{12}$ In HUVECs MVA/T7RP-mediated expression was also detected for up to 4 days with peak expression occurring at 1-2 days
(Figure 4c). To determine whether such transient kinetics were a property of the MVA/T7RP system in general or of HUVECs in particular, the electroporation and infection conditions were applied to primary rabbit microvascular endothelial cells. These cells showed CAT expression that remained high for up to 2 weeks (data not shown).

\section{Magnetic immunoaffinity cell sorting}

The developed electroporation and infection techniques were coupled with a magnetic immunoaffinity cell sorting procedure to isolate the transfected HUVEC population. ${ }^{19,20}$ The alpha subunit of the interleukin-2 cell surface receptor (IL2R $\alpha$ ) was used as a sorting target. After square-wave Petri dish electroporation of PT7/IL2R $\alpha$ DNA and infection with MVA/T7RP, distinctive antiIL2R $\alpha$-directed magnetic bead binding was observed in IL2R $\alpha$ - (Figure 5) but not mock-transfected (data not shown) cells as detected by light microscopy. The percent recovery of radiolabeled IL2R $\alpha$-transfected HUVECs isolated by anti-IL2R $\alpha$ magnetic immunoaffinity cell sorting was $0.9 \pm 0.3 \%$ and $15.9 \pm 2.0 \%(n=3)$ following 350 and $450 \mathrm{~V}$ pulses, respectively, for $20 \mathrm{~ms}$. This agreed with microscopic observations.

The PT7/IL2R $\alpha$ gene was also used to prepare a bicistronic IL2R $\alpha$ /CAT DNA expression unit under PT7 control, a representation of which is shown in Figure 6a. Use of this DNA should result in expression of both the sorting target IL $2 \mathrm{R} \alpha$ and the reporter gene CAT from the same vector. The bicistronic design was possible because of the internal ribosome entry site (IRES) preceding each gene in the IL2R $\alpha /$ CAT expression unit. After squarewave, Petri dish electroporation of PT7/IL2R $\alpha / C A T$ DNA and MVA/T7RP infection, HUVECs underwent anti-IL2R $\alpha$-targeted magnetic immunoaffinity cell sorting to separate the transfected from the nontransfected cell 


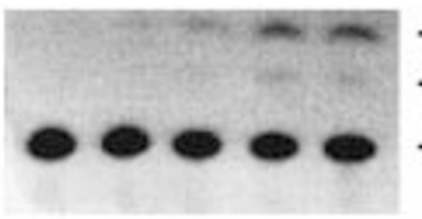

$-\mathrm{CM}-3 \mathrm{AC}$

$-\mathrm{CM}-1 \mathrm{AC}$

$-\mathrm{CM}$

\section{$\begin{array}{lllll}0 & 1 & 5 & 10 & 15\end{array}$}

MVATT7RP (m. o. i.)

b

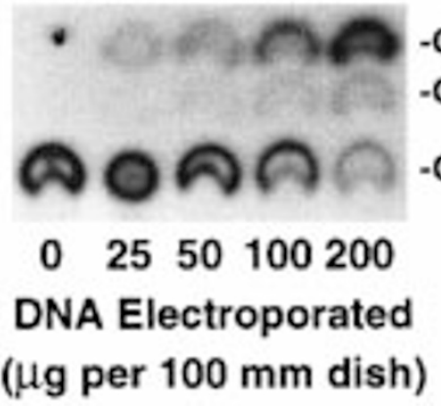

C

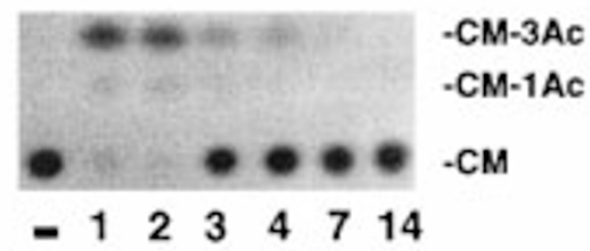

Days Post-Transfection

Figure 4 Characterization of infection and transfection conditions. (a) HUVECs were square-wave electroporated with $50 \mu \mathrm{g}$ of PT7/CAT DNA using a Petri dish electrode at $350 \mathrm{~V}$ for $20 \mathrm{~ms}$. The cells were then infected with the indicated levels of MVA/T7RP. Twenty-four hours after transfection, HUVECS were lysed and analyzed for CAT activity. The resultant autoradiogram of separated chloramphenicol products is shown. (b) HUVECs were transfected with the indicated amounts of PT7/hCAT DNA using a Petri dish electrode at $350 \mathrm{~V}$ for $20 \mathrm{~ms}$ and then infected with 10 MOI of MVA/T7RP. Cell extracts were analyzed for CAT activity as described. (c) HUVECs were transfected with $50 \mu g$ of PT7/CAT DNA using a Petri dish electrode at $350 \mathrm{~V}$ for $20 \mathrm{~ms}$ and then infected with 10 MOI of MVA/T7RP. At the indicated times, cells were harvested and extracts were analyzed for CAT activity. The resultant autoradiograph of TLC-separated reaction products is shown. $\left[{ }^{14} \mathrm{C}\right]$ chloramphenicol $(\mathrm{CM})$, $\left[{ }^{14} \mathrm{C}\right]$ chloramphenicol 1-monoacetate $(\mathrm{CM}-1 \mathrm{Ac}) ;\left[{ }^{14} \mathrm{C}\right]$ chloramphenicol 3-monoacetate (CM-3Ac).

populations. Expression from the bicistronic IL2R $\alpha /$ CAT DNA sequence and fidelity of the sorting technique was verified by over 100-fold enrichment of CAT activity in the positively sorted cell population (Figure 6b).

To determine whether such bicistronic expression was applicable to more physiological genes besides the common reporter CAT, a similar strategy was used to achieve expression of bicistronic T7 promoter-directed IL2R $\alpha$ /FLAG-tagged retinoblastoma protein family member sequences. Expression of each of FLAG-tagged $\mathrm{pRb}, \mathrm{p} 107$ and $\mathrm{pRb} 2 / \mathrm{p} 130$ proteins could be detected by anti-FLAG immunoblotting in the anti-IL2R $\alpha$-targeted, magnetic immunoaffinity cell sorted HUVEC population (Figure 7).

\section{Expression of green fluorescent protein}

To examine whether the established electroporation and infection conditions were sufficient for expression of

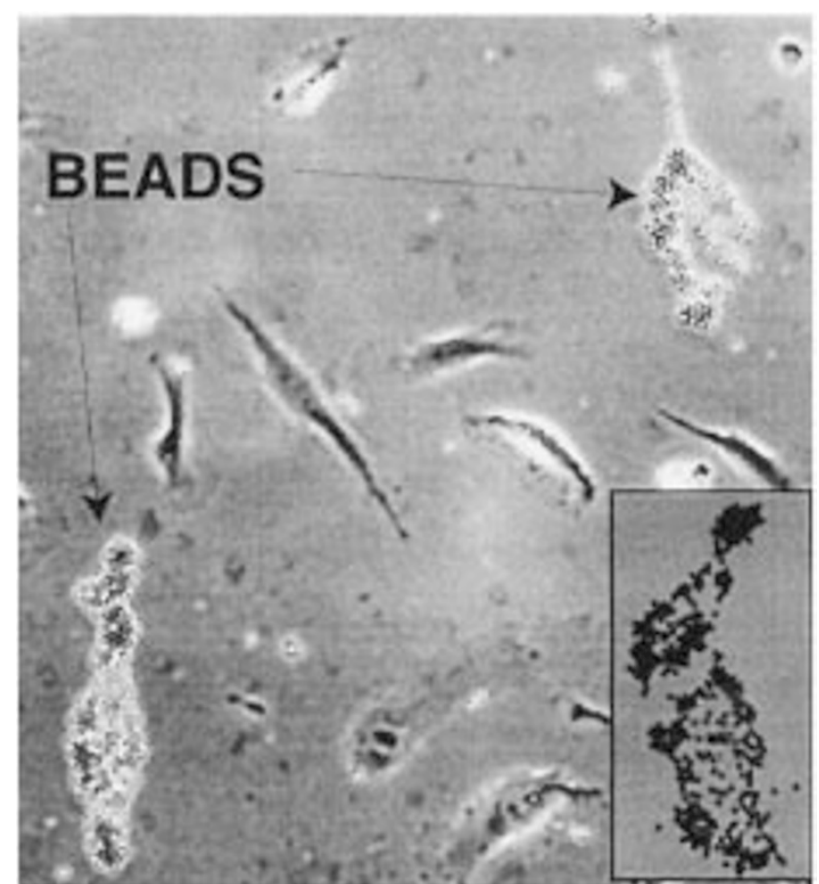

Figure 5 Anti-IL2R $\alpha$-directed magnetic immunoaffinity cell sorting. HUVECs were electroporated with PT7/IL2R $\alpha$ DNA and infected with $M V A / T 7 R P$. IL2R $\alpha$ expression was detected $16 \mathrm{~h}$ later by incubating the cells with mouse anti-IL2R $\alpha$ antibody and goat anti-mouse antibody-conjugated magnetic beads. The cells were imaged in normal phase. The arrows indicate HUVECs that bound beads. The inset shows two IL2R $\alpha$ expressing HUVECs imaged in bright field to enhance the appearance of the bound magnetic beads.

a

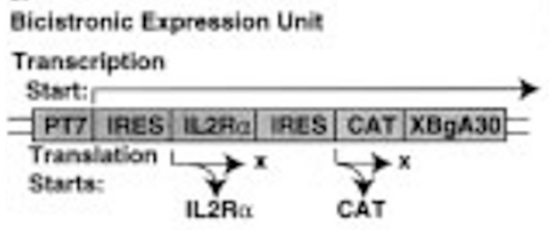

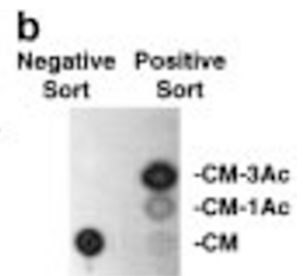

Figure 6 Fidelity of magnetic immunoaffinity cell sorting. (a) A T7 promoter (PT7)-directed expression unit was prepared containing the IL2R $\alpha$ and CAT genes each preceded by an internal ribosome entry site (IRES) from the encephalomyocarditis virus. A 3' untranslated region derived from the Xenopus laevis $\beta$ globin gene followed by a 30 residue adenine tract completes the expression unit as shown. (b) HUVECs were electroporated with a DNA vector containing the T7 expression unit shown in panel a. Transfected and infected HUVECs that bound beads (positive sort) were separated from those that did not (negative sort) using antiIL2R $\alpha$-targeted magnetic affinity cell sorting. Normalized protein from each population was analyzed for CAT activity. Unmodified $\left[{ }^{14} \mathrm{C}\right]$ chloramphenicol $(C M)$ was separated from $\left[{ }^{14} \mathrm{C}\right]$ chloramphenicol 1-monoacetate $(C M-1 A c)$ and $\left[{ }^{14} \mathrm{C}\right]$ chloramphenicol 3-monoacetate $(C M-3 A c)$ by thin-layer chromatography and the resultant autoradiograph is shown.

genes other than the reporter gene CAT and the sorting marker IL2R $\alpha$, a T7 expression unit was prepared for overexpression of green fluorescent protein (GFP). ${ }^{21}$ This PT7/GFP DNA vector was transfected into HUVECs using the established electroporation and MVA/T7RP infection conditions. Transfected, viable HUVECs were readily evident as detected by fluorescence microscopy (Figure 8). Interestingly, HUVEC GFP expression appeared somewhat nucleophilic. 

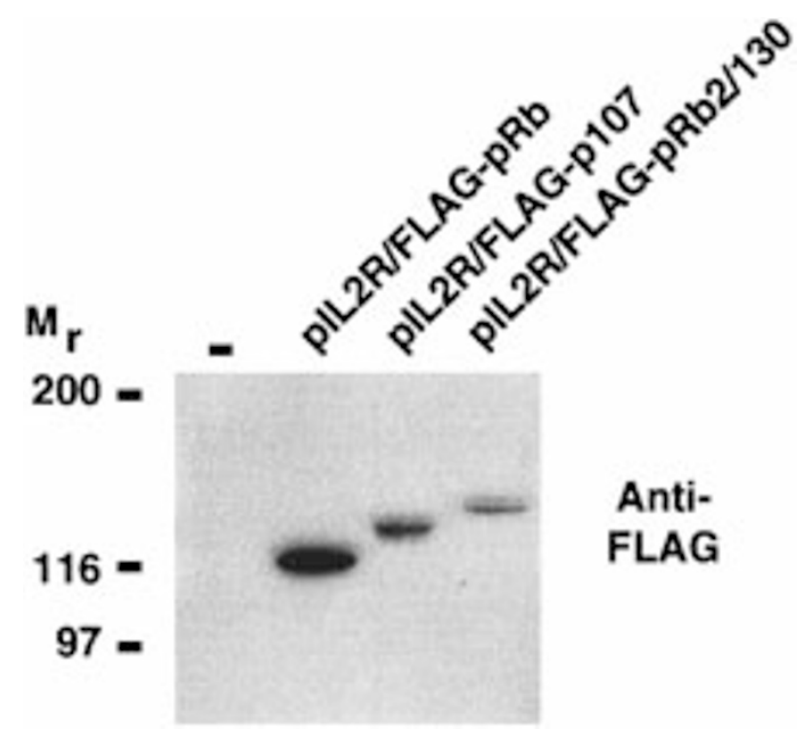

Figure 7 Expression of bicistronic IL2R/FLAG-tagged retinoblastoma protein family members. T7 promoter (PT7)-directed expression units were prepared similar to that shown in Figure 5a with either FLAG-tagged $p R b, p 107$ or $p R b 2 / p 130$ genes in place of the CAT gene. MVA/T7RPinfected HUVECs were electroporated with $50 \mu \mathrm{g}$ of PT7/IL2R $\alpha$ DNA vector (-) or PT7/IL2R $\alpha / F L A G-p R b, \quad P T 7 / I L 2 R \alpha / F L A G-p 107$, PT7/IL2R $\alpha / F L A G-p R b 2 / p 130$. Twenty-hour hours after transfection, the cells were harvested and equivalent amounts of extract were analyzed by SDS-PAGE and immunoblotting with anti-FLAG antibodies. Immunoreactive proteins were detected by chemiluminescence. Calculated molecular weights for FLAG-pRb, FLAG-p107 and FLAG-pRb2/p130 are 107, 122 and $130 \mathrm{kDa}$, respectively.

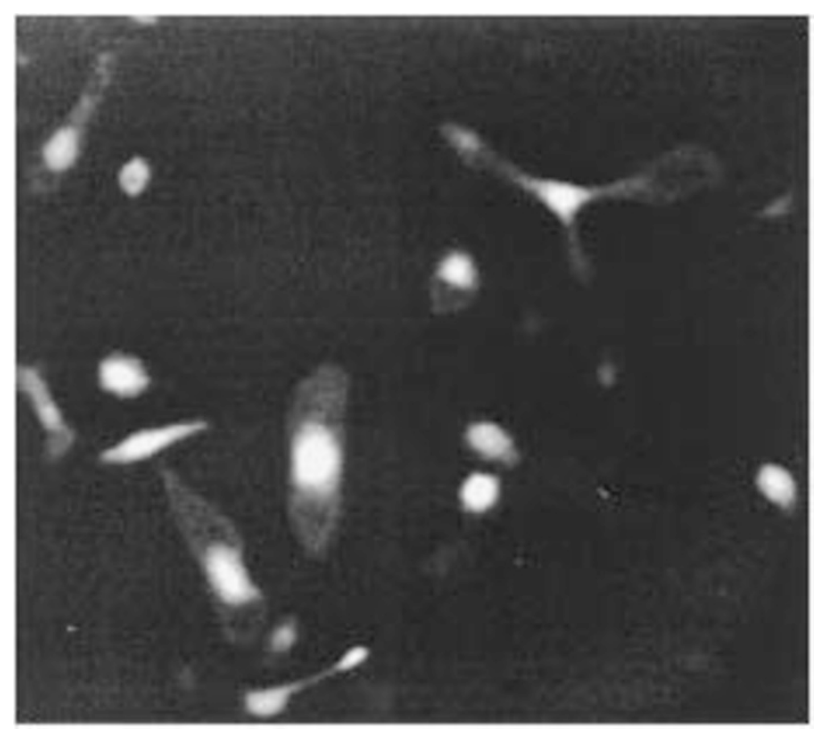

Figure 8 Expression of green fluorescent protein in HUVECs. Cells were tansfected with $50 \mu \mathrm{g}$ of PT7/GFP DNA using a Petri dish electrode and infected with 10 MOI of MVA/T7RP. Twenty four hours later cells were washed and visualized by epifluorescence microscopy.

\section{Overexpression of a panel of proteins}

To examine further use of the expression system with a variety of genes, T7 expression vectors were prepared containing either the cyclin-dependent kinase inhibitors $\mathrm{p} 21^{c i p 1}$ or $\mathrm{p} 27^{k i p 1}$, the protein tyrosine kinase $\mathrm{pp} 60^{\mathrm{c}-s r c}$, members of the retinoblastoma family pRb, p107 and
$\mathrm{pRb} 2 / \mathrm{p} 130$, and fibroblast growth factor (FGF)-1. ${ }^{22-28}$ immunoblotting after using the established electroporation and infection conditions (Figure 9). Each product migrated at the expected apparent molecular weight and was specifically observed in the sample-transfected HUVECs (Figure 9).

\section{Discussion}

The MVA/T7RP expression system and a cellular sorting method were coupled with square-wave electroporation using a Petri dish electrode for gene expression in primary cultures of HUVECs. Results showed: (1) high, but transient, transgene expression after transfection of $\mathrm{T} 7$ promoter-directed target genes into MVA/T7RP-infected cells; (2) selective isolation of the transfected cell population by magnetic immunoaffinity cellular sorting against a co-expressed cell surface protein; and (3) successful application of a Petri dish electrode with resultant increased HUVEC viability and overall expression. The success of the system was manifested by the overexpression of a variety of genes, including those for nonreporter proteins, in HUVECs.

The nonreplicating MVA/T7RP recombinant vaccinia virus used in this study provides high T7RP-directed expression with diminished cytopathic effects. ${ }^{12-14}$ The MVA strain has been used successfully as an expression vehicle for the immunotherapeutic treatment of infectious disease and cancer in animal models and was successfully safety tested in humans. ${ }^{29-34}$ In the approach described here, a T7 promoter-directed transgene is introduced by transfection and the T7 RNA polymerase is expressed by the virus. This obviates the need to prepare recombinant viruses for each desired transgene.

Using this approach, gene expression was examined

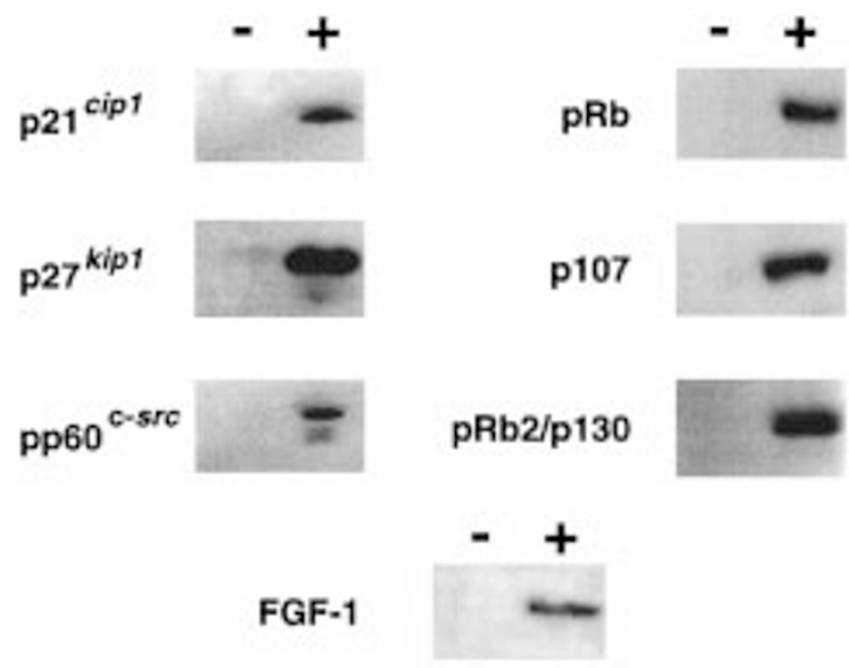

Figure 9 Immunoblots of proteins expressed in HUVECs using the electroporation and infection conditions. Cells were transfected with $50 \mu \mathrm{g}$ of an empty DNA vector (-) or the same vector with the indicated genes under control of a T7 promoter (+). The transfected HUVECs were infected with 10 MOI of MVA/T7RP. Twenty-four hours after transfection, the cells were harvested and equivalent amounts of extract were analyzed by SDS-PAGE and immunoblotting. Immunoreactive proteins were detected by chemiluminescence and the resultant autoradiographs are shown. 
after varying the voltage applied during electroporation, the MVA/T7RP infection level and PT7/target gene DNA concentration. A single 350 or $450 \mathrm{~V}$ pulse for $20 \mathrm{~ms}$ with $50 \mu \mathrm{g}$ of DNA in $10 \mathrm{ml}$ electroporation buffer and viral adsorption with $10 \mathrm{MOI}$ was sufficient for readily detectable expression of CAT activity using the Petri dish electrode. Higher CAT expression was achieved by increasing the voltage, the amount of transfected PT7containing DNA or the MVA/T7RP infection level within tested limits.

Use of the MVA/T7RP system with the Petri dish electrode resulted in higher overall CAT expression and increased numbers of viable cells relative to a cuvette electrode using respective optimized electroporation conditions. Expression achieved was at least 10000 -fold higher than that with CMV/CAT expression based on the dilution of lysates required for CAT assay.

Optimizing any expression method involves maximizing expression balanced against resultant cytotoxic effects. Petri dish electroporation resulted in a higher number of viable cells compared with cuvette electroporation after application of the MVA/T7RP system. The combination of infection and electroporation appeared somewhat synergistic in decreasing the number of viable cells that was less severe for the Petri dish electrode. Decreases in the number of viable cells may be due to cell death or, since untreated cells exhibited proliferation, inhibition of cell growth. The level of cell viability achieved here is similar or improved over those reported for HUVECs and other cell types using comparable methods. $^{1,35}$

The additional cellular manipulation required of the cuvette electrode in addition to viral infection may be detrimental. Consistent with this, similar viability was observed regardless of electrode type during nonviral CMV/CAT expression.

The improved viability and resultant increased overall expression in addition to the minimization of HUVEC perturbation justifies use of the Petri dish electrode with the MVA/T7RP system. The short transfection (ms) time and avoidance of chemicals typical of electroporation contrast with the extended transfection times of $6 \mathrm{~h}$ or more for maximal DNA uptake during lipofections ${ }^{36}$ that are often accompanied by cytotoxic effects. Petri dish electroporation is further desirable as it also avoids disruption of the cell monolayer inherent to cuvette electroporation. This may maintain cellular stability that is more reflective of the state of endothelial cells in vivo.

Higher applied voltages appear necessary to achieve adequate expression for Petri dish versus cuvette electroporation. This may be required to overcome a larger voltage drop between the Petri dish electrode and the cells that is caused by a larger buffer volume and increased target area relative to a cuvette electrode. The lack of significant cytotoxic effects even though using relatively higher voltages with a Petri dish electrode may also be partly explained by a larger voltage drop.

The kinetics of expression were measured and CAT activity was detectable from 1 to 4 days. Highest expression was at 1-2 days. The subsequent decrease in expression may be due to transience or dilution of the expression components (PT7 DNA, T7RP enzyme), a loss of transfected cells, or loss of CAT protein over time. Importantly, extended CAT expression for up to 2 weeks was achieved in primary cultures of rabbit microvascular endothelial cells. This suggests that the T7 expression kinetics are cell-type specific and highlights the magnitude of the differences in expression commonly observed between HUVECs and other types of cells.

Expression of the cell surface protein IL2R $\alpha$ was used as a molecular target to isolate the transfected population by magnetic immunoaffinity cell sorting. Transfection efficiency was quantified at $0.9 \%$ and $15.9 \%$ at 350 or $450 \mathrm{~V}$, respectively, for $20 \mathrm{~ms}$. The increase in transfection efficiency as a function of voltage potentially explains the overall increase in CAT expression caused by increasing the voltage applied.

As the number of transfected cells is in the minority, the ability to isolate the transfected cell population enables enrichment for cells harboring the transgene. Cellular sorting overcomes low transfection efficiencies and allows analysis in a background of diminished nontransfected cells and endogenous gene products. Here, isolation of the transfected cell population was achieved with high recovery and fidelity by anti-IL2R $\alpha$-targeted magnetic immunoaffinity cell sorting. A bicistronic expression unit allowed production of both the sorting marker IL2R $\alpha$ and the reporter gene CAT from the same DNA vector. This obviated the necessary cellular colocalization of both genes if expressed from two different vectors and potentially resulted in improved sorting efficiency. Accordingly, CAT activity expressed from the bicistronic T7 promoter-directed IL2R $\alpha$ /CAT expression unit was specifically segregated into the positively sorted cell population.

A similar strategy was used to achieve expression of DNA sequences containing bicistronic T7 promoterdirected IL2R $\alpha /$ FLAG-tagged retinoblastoma protein family members. Expression of each of FLAG-tagged $\mathrm{pRb}, \mathrm{p} 107$ and $\mathrm{pRb} 2 / \mathrm{p} 130$ proteins could be detected by anti-FLAG immunoblotting in the anti-IL2R $\alpha$-targeted, magnetic immunoaffinity cell sorted HUVEC population. Thus, bicistronic IL2R $\alpha$ /target gene expression coupled with the sorting method appears feasible for efficient and specific isolation of the expressing cell population for physiological genes in addition to the reporter gene CAT.

The electroporation and infection conditions chosen were sufficient for the overexpression of a variety of proteins. The fluorophore GFP was readily detected and appeared nucleophilic in HUVECs. In addition, a panel of ectopically expressed proteins was detected by immunoblotting and included the cyclin-dependent kinase inhibitors $\mathrm{p} 21^{\mathrm{cip} 1}$ and $\mathrm{p} 27^{\mathrm{kip} 1}$, the protein tyrosine kinase pp60 ${ }^{\mathrm{c}-s r c}$, members of the retinoblastoma family pRb, p107 and $\mathrm{pRb} 2 / \mathrm{p} 130$, and the angiogenic polypeptide FGF-1. Interestingly, although the human proteins expressed from the ectopic genes were readily detected, their endogenous counterparts were not with the number of HUVECs analyzed and under the exposure conditions used. This appears indicative of the superphysiological expression levels achieved by the coupled viral T7 RNA polymerase system which can achieve levels as high as $10 \%$ of cellular protein. ${ }^{18}$

Use of the MVA/T7RP system in HUVECs appears to favor experimental and therapeutic applications requiring extremely high expression occurring during transient expression periods. Possible applications of this system include biochemical analysis of the ectopically expressed gene or affected molecules and short-term cell cycle analysis. The high expression afforded this system would 
also appear optimal for high-output production of secreted ectopic proteins from endothelial cells or overexpression of proteins from endothelial cells for subsequent purification purposes. This system may be desirable for the application of knockout, dominant negative or antisense technology where ultra-high expression is required. In addition, the system may be useful in rapidly screening expression of different genes as the preparation of recombinant viruses for each desired transgene is not required of this approach. Use of the nonreplicating vaccinia virus/T7 RNA polymerase expression system with square wave, Petri dish electroporation appears feasible for certain applications for the study of endothelial cells by gene transfer.

\section{Materials and methods}

\section{Human umbilical vein endothelial cells}

Human umbilical cords were obtained through the Cardeza Foundation for Hematologic Research, Thomas Jefferson University. HUVECs were isolated as described ${ }^{37}$ and grown on human fibronectin-coated plates $\left(2 \mu \mathrm{g} / \mathrm{cm}^{2}\right)^{38,39}$ in complete growth medium (M199 (Mediatech, Herndon, VA, USA) containing 10\% (v/v) fetal bovine serum (Hyclone, Logan, UT, USA), $100 \mu \mathrm{g} / \mathrm{ml}$ endothelial cell growth supplement, ${ }^{40} 10 \mathrm{U} / \mathrm{ml}$ heparin (Elkins-Sinn, Cherry Hill, NJ, USA) and antibioticantimycotic) in $95 \%$ humidified air, $5 \% \mathrm{CO}_{2}$ at $37^{\circ} \mathrm{C}$. The endothelial identity of the cells was verified by positive immunostaining for the endothelial cell-specific marker PECAM-1. The number of viable HUVECs was measured after incubation in a 1:1 (v/v) mixture of resuspended cells and $0.4 \%$ trypan blue. Unstained HUVECs were counted using a hemacytometer.

\section{Plasmid construction}

DNA vector reagents containing $\mathrm{T} 7$ expression units were prepared using standard procedures with the pMINI plasmid as the vector backbone. ${ }^{11}$ Each T7 expression unit contained the cDNA of interest with flanking $5^{\prime}$ and $3^{\prime}$ structures that enhance the expression of T7 transcripts in eukaryotic cells. The region flanking the $5^{\prime}$ end of the gene consisted of a T7 promoter (PT7) for T7 RNA polymerase-mediated expression and an internal ribosome entry site (IRES) derived from the encephalomyocarditis virus for cap-independent translation. ${ }^{41}$ The region flanking the $3^{\prime}$ end of the transgene contained the untranslated region from the Xenopus laevis $\beta$ globin gene followed by 30 adenine residues $\left(\mathrm{A}_{30}\right){ }^{42}$ Genes chosen for expression were the chloramphenicol acetyltransferase enzyme (CAT), green fluorescent protein (GFP, green lantern 'humanized' GFP, GIBCO/Life Technologies, Rockville, MD, USA), p21 cip1, $\mathrm{p} 27^{k i p 1}$, pp $60^{\mathrm{c}-s r c}$, pRb, p107, pRb2/p130 and FGF-1. ${ }^{21-28,43}$

A T7 expression unit was also prepared containing the alpha subunit of the interleukin-2 receptor (IL2R $\alpha){ }^{44}$ IL2R $\alpha$ expression acts as a surface marker for anti-IL2R $\alpha$ directed magnetic immunoaffinity cellular sorting. The PT7/IL2R $\alpha$ DNA was then used to prepare bicistronic PT7/IL2R $\alpha /$ CAT (see Figure 6a) and PT7/IL2R $\alpha$ / retinoblastoma family member expression units.

\section{Transfection and infection}

Transfections were conducted by square-wave electroporation using a T820 Electrosquare Porator

(BTX/Genetronics, San Diego, CA, USA). HUVECs were seeded at 400000 cells per $100 \mathrm{~mm}$ dish in complete growth medium the day preceding the transfection and were at sub-confluence at the time of infection and transfection (see Figure 5 for the approximate degree of confluence). Cells were washed once with filter-sterilized electroporation buffer $(20 \mathrm{~mm}$ Hepes, pH 7.05, $137 \mathrm{~mm}$ $\mathrm{NaCl}, 5 \mathrm{~mm} \mathrm{KCl}, 0.7 \mathrm{~mm} \mathrm{Na} \mathrm{HPO}_{4} 6 \mathrm{~mm}$ dextrose, $1 \mathrm{mg} / \mathrm{ml}$ bovine serum albumin ${ }^{45}$ ) and incubated for $15 \mathrm{~min}$ at room temperature in $2 \mathrm{ml}$ electroporation buffer with $50 \mu \mathrm{g}$ DNA or other amount as stated. DNA was purified either by $\mathrm{CsCl}$-double band ultracentrifugation or by anion exchange chromatography (MaxiPrep, 5 Prime $\rightarrow 3$ Prime, Boulder, CO, USA). The electroporation volume was increased to $10 \mathrm{ml}$ and placed in contact with a 100-mm sized, 2-mm gap Petri dish electrode with stainless steel plates (BTX/Genetronics). The electrode plates were cleaned by water-bath sonication and $70 \%$ ethanol rinse. The complete electrode was sterilized by exposure to ethylene oxide. After the indicated electroporation conditions were applied, the DNA solution was aspirated and the nonreplicating vaccinia virus strain modified vaccinia virus Ankara expressing T7RP (MVA/T7RP) (a gift of L Wyatt and B Moss, National Institutes of Health, Bethesda, MD, USA) was added at indicated infection levels in $2 \mathrm{ml}$ reduced serum medium (OPTI-MEM I; Life Technologies) for $30 \mathrm{~min}$ at $37^{\circ} \mathrm{C}$. After the adsorption period, the viral solution was removed and cells were placed in complete growth medium for 16-24 h unless indicated otherwise.

\section{CAT assays}

Gene expression was measured in the cell lysates by assaying for CAT activity using radiolabeled chloramphenicol as substrate. Analysis was by thin-layer chromatography (TLC). ${ }^{46}$ Unless otherwise stated, extracts were prepared from transfected HUVECs 16-24 h after transfection. For quantification, if conversion of greater than $80 \%$ of chloramphenicol occurred, the assay was considered out of the linear range and lysates were diluted appropriately for analysis.

\section{Magnetic immunoaffinity cell sorting}

The cell surface expression of IL2R $\alpha$ has been used as a target for subsequent isolation of transfected cells by magnetic immunoaffinity cell sorting. ${ }^{19,20}$ HUVECs were electroporated with either PT7/IL2R $\alpha$ DNA or a bicistronic PT7/IL2R $\alpha /$ CAT DNA and infected with MVA/T7RP. The following day the HUVECs were incubated in OPTI-MEM with magnetic beads (M-5, Dynal, Lake Success, NY, USA) coated with goat anti-mouse antibodies directed against mouse anti-IL2R $\alpha$ antibodies (Upstate Biotechnology, Lake Placid, NY, USA). The cells were lifted from the plates and subjected to a magnetic force to separate the cells that bound beads from those that did not as described previously..$^{19,20}$

To determine transfection efficiency the anti-IL2R $\alpha$ directed magnetic immunoaffinity cell sorting procedure was applied to PT7/IL2R $\alpha$-transfected and infected HUVECs that were then radiolabeled with $0.1 \mathrm{mCi} / \mathrm{ml}$ ${ }^{14} \mathrm{C}$-thymidine (New England Nuclear, Boston, MA, USA). Quantification was by scintillation counting of the trichloroacetic acid-precipitable material from the sorted relative to the total population. Transfection efficiency was expressed as a percentage of the counts sorted rela- 
tive to the total counts. HUVECs transfected with an empty pMINI vector served as a negative control.

\section{Fluorescence microscopy}

Green fluorescent protein (GFP) isolated from the bioluminescent jellyfish Aequorea victoria absorbs light and fluoresces without additional cofactors or substrates and is a useful reporter for gene expression in living cells. ${ }^{21}$ GFP expression was detected by placing the tissue culture plate with cells and medium under an Axiovert 100A inverted epifluorescence microscope (Carl Zeiss, Thornwood, NY, USA) with a HB100W mercury short arc lamp (Atto Instruments, Rockville, MD, USA). The filter set was a standard fluorescein isothiocyanate set (Chroma Technologies, Brattleboro, VT, USA) with excitation and emission centered at $480 \mathrm{~nm}$ (range 460-500 nm) and $535 \mathrm{~nm}$ (range 510-560 nm). Image acquisition was conducted with a CCD video camera (Grundig, Fürth, Germany), Scion Frame Grabber hardware (Applied Scientific Instrumentation, Eugene, OR, USA) and NIH Image software.

\section{Immunoblotting}

Transfected HUVECs were removed from tissue culture dishes, isolated by centrifugation and lysed in $66 \mathrm{~mm}$ Tris- $\mathrm{HCl}, \mathrm{pH} 6.8$ containing 2\% (w/v) SDS and $10 \mathrm{~mm}$ EDTA for $10 \mathrm{~min}$ at $95^{\circ} \mathrm{C}$. Equal amounts of lysate were resolved by sodium dodecyl sulfate/polyacrylamide gel electrophoresis under reducing conditions and transferred to nitrocellulose membranes (Amersham, Piscataway, NJ, USA). The membranes were incubated with TBS/Tween $20(10 \mathrm{~mm}$ Tris-HCl, pH 8.0, $150 \mathrm{~mm} \mathrm{NaCl}$, $0.05 \%(\mathrm{v} / \mathrm{v})$ Tween 20$)$ containing $5 \%(\mathrm{w} / \mathrm{v})$ nonfat dry milk to block nonspecific binding. The membranes were incubated with primary antibody in TBS/Tween 20 containing $1 \%(\mathrm{w} / \mathrm{v})$ nonfat dry milk, washed with TBS/Tween 20, and then incubated with horseradish peroxidase-conjugated secondary antibody (Kirkegaard \& Perry Laboratories, Gaithersburg, MD, USA; or Jackson Laboratories, Bar Harbor, ME, USA) in TBS/Tween 20 containing $1 \%(\mathrm{w} / \mathrm{v})$ nonfat dry milk. After washing with TBS/Tween 20, immunoreactive proteins were visualized using enhanced chemiluminescence detection (Pierce, Rockford, IL, USA) with exposure on to autoradiographic film. Primary antibodies were anti-IL2R $\alpha$ and anti-aFGF (Upstate Biotechnology), anti-FLAG (Kodak, New Haven, CT, USA), anti-p27 ${ }^{k i p 1}$ (Transduction Laboratories, Lexington, KY, USA), anti-pRb (Pharmingen, San Diego, CA, USA) and anti-p21 cip1, anti-p107, antipRb2/p130 and anti-pp60 ${ }^{\text {c-src }}$ (Santa Cruz Biotechnology Santa Cruz, CA, USA).

\section{Acknowledgements}

This work was supported by a National Research Council Research Associateship to Kurt A Engleka while a postdoctoral fellow at the National Institutes of Health, institutional support from Thomas Jefferson University and by a grant from the American Heart Association. The authors would like to thank Donald J Fujita, University of Calgary, Vasily V Ogryzko, National Institutes of Health, and Antonio Giordano, Thomas Jefferson University for supplying plasmids, Linda S Wyatt and Bernard Moss, National Institutes of Health, for MVA/T7RP recombinant virus, Marilyn Woolkalis, Thomas Jefferson
University for HUVECs and P Macke Consigny, Thomas Jefferson University for the use of a fluorescence microscope and imaging system and rabbit microvascular endothelial cells.

\section{References}

1 Schwachtgen J-L, Ferreira V, Meyer D, Kerbiriou-Nabias D. Optimization of the transfection of human endothelial cells by electroporation. BioTechniques 1994; 17: 882-887.

2 Tanner FC, Carr DP, Nabel GJ, Nabel EG. Transfection of human endothelial cells. Cardiovascular Res 1997; 35: 522-528.

3 Teifel M, Heine L-T, Milbredt S, Friedl P. Optimization of transfection of human endothelial cells. Endothelium 1997; 5: 21-35.

4 Edgell C-JS, Curiel DT, Hu P-C, Marr HS. Efficient gene transfer to human endothelial cells using DNA complexed to adenovirus particles. BioTechniques 1998; 25: 264-272.

5 Fife $\mathrm{K}$ et al. Endothelial cell transfection with cationic liposomes and herpes simplex-thymidine kinase mediated killing. Gene Therapy 1998; 5: 614-620.

6 Wilson DB, Dorfman DM, Orkin SH. A nonerythroid GATAbinding protein is required for function of the human preproendothelin-1 promoter in endothelial cells. Mol Cell Biol 1992; 10 4854-4862.

7 Nabel EG, Nabel GJ. Complex models for the study of gene function in cardiovascular biology. Ann Rev Physiol 1994; 56: 741-761.

8 Inaba $\mathrm{M}$ et al. Retroviral gene transfer: effects on endothelial cell phenotype. J Surg Res 1998; 78: 31-36.

9 de Martin R, Raidl M, Hofer E, Binder BR. Adenovirus-mediated expression of green fluorescent protein. Gene Therapy 1997; 4: 493-495.

10 Riccioni $\mathrm{T}$ et al. Adenovirus-mediated wild-type p53 overexpression inhibits endothelial cell differentiation in vitro and angiogenesis in vivo. Gene Therapy 1998; 5: 747-754.

11 Engleka KA, Lewis EW, Howard BH. Mechanisms of replication-deficient vaccinia virus/T7 RNA polymerase hybrid expression; effect of T7 RNA polymerase levels and alphaamanitin. Virology 1998; 243: 331-339.

12 Sutter G, Moss B. Nonreplicating vaccinia vector efficiently expresses recombinant genes. Proc Natl Acad Sci USA 1992; 89: 10847-10851.

13 Sutter G, Olmann M, Erfle V. Non-replicating vaccinia vector efficiently expresses bacteriophage T7 RNA polymerase. FEBS Lett 1995; 371: 9-12.

14 Wyatt LS, Moss B, Rozenblatt S. Replication-deficient vaccinia virus encoding bacteriophage T7 RNA polymerase for transient gene expression in mammalian cells. Virology 1995; 210: 202-205.

15 Moss B et al. New mammalian expression vectors. Nature 1990; 348: 91-92.

16 Muramatsu T, Nakamura A, Park H-M. In vivo electroporation: a powerful and convenient means of nonviral gene transfer to tissues of living animals. Int I Mol Med 1998; 1: 55-62.

17 Nathwani AC et al. Efficient gene transfer into human umbilical vein endothelial cells allows functional analysis of the human tissue factor gene promoter. Br J Haematol 1994; 88: 122-128.

18 Elroy-Stein O, Moss B. Cytoplasmic expression system based on constitutive synthesis of bacteriophage T7 RNA polymerase in mammalian cells. Proc Natl Acad Sci USA 1990; 87: 6743-6747.

19 Giordano $\mathrm{T}$ et al. Isolation of a population of transiently transfected quiescent and senescent cells by magnetic affinity cell sorting. Exp Cell Res 1991; 192: 193-197.

20 Padmanabhan $R$ et al. Purification of transiently transfected cells by magnetic affinity cell sorting. Anal Biochem 1988; 170: 341348.

21 Prasher DC et al. Primary structure of the Aequorea victoria green-fluorescent protein. Gene 1992; 111: 229-233.

22 Harper JW et al. The p21 Cdk-interacting protein Cip1 is a potent inhibitor of G1 cyclin-dependent kinases. Cell 1993; 75: 805-816. 
23 Jaye $\mathrm{M}$ et al. Human endothelial cell growth factor: cloning nucleotide sequence, and chromosome localization. Science 1986; 233: 541-545.

24 DeCaprio JA et al. SV40 large tumor antigen forms a specific complex with the product of the retinoblastoma susceptibility gene. Cell 1988; 54: 275-283.

25 Ewen ME, Xing Y, Lawrence JB, Livingston D. Molecular cloning, chromosomal mapping, and expression of the cDNA for p107, a retinoblastoma gene product-related protein. Cell 1991; 66: 1155-1164.

26 Mayol $X$ et al. Cloning of a new member of the retinoblastoma gene family (pRb2) which binds to the E1A transforming domain. Oncogene 1993; 8: 2561-2566.

27 Tanaka A et al. DNA sequence encoding the amino-terminal region of the human c-src protein: implications of sequence divergence among src-type kinase oncogenes. Mol Cell Biol 1987; 7: 1978-1983.

28 Polyak $\mathrm{K}$ et al. Cloning of $\mathrm{p} 27^{\mathrm{Kip} 1}$, a cyclin-dependent kinase inhibitor and a potential mediator of extracellular antimitogenic signals. Cell 1994; 78: 59-66.

29 Moss B et al. Host range restricted, non-replicating vaccinia virus vectors as vaccine candidates. Adv Exp Med Biol 1996; 397: 7-13.

30 Bender BS et al. Oral immunization with a replication-deficient recombinant vaccinia virus protects mice against influenza. J Virol 1996; 70: 6418-6424.

31 Sutter G et al. A recombinant vector derived from the host range-restricted and highly attenuated MVA strain of vaccinia virus stimulates protective immunity in mice to influenza virus. Vaccine 1994; 12: 1032-1040.

32 Schneider $\mathrm{J}$ et al. Enhanced immunogenicity for CD8+ T cell induction and complete protective efficacy of malaria DNA vaccination by boosting with modified vaccinia virus Ankara. Nature Med 1998; 4: 397-402.

33 Hanke $T$ et al. Immunogenicities of intravenous and intramuscular administrations of modified vaccinia virus Ankara-based multi-CTL epitope vaccine for human immunodeficiency virus type 1 in mice. J Gen Virol 1998; 79: 83-90.

34 Mayr A, Hochstein-Mintzel V, Stickl H. Passage history, proper- ties, and applicability of the attenuated vaccinia virus strain MVA. Infection 1973; 3: 6-14.

35 van den Hoff MJB, Moorman AFM, Lamers WH. Electroporation in 'intracellular' buffer increases cell survival. Nucleic Acids Res 1992; 20: 2902

36 Zabner J et al. Cellular and molecular barriers to gene transfer by a cationic lipid. J Biol Chem 1995; 270: 18997-19007.

37 Jaffe EA, Nachman RL, Becker CG, Minick CR. Culture of human endothelial cells derived from umbilical veins. J Clin Invest 1973; 52: 2745-2746.

38 Vuento M, Vaheri A. Purification of fibronectin from human plasma by affinity chromatography under non-denaturing conditions. Biochem J 1979; 183: 331-337.

39 Regnault V, Rifat C, Stoltz JF. Affinity purification of human plasma fibronectin on immobilized gelation. J Chromatogr 1988; 432: 93-102.

40 Maciag T, Weinstein R, Preparation of endothelial cell growth factor. In: Barnes DW, Sirbasku DA, Sato GH (eds). Methods for Preparation of Media, Supplements, and Substrata for Serum-free Animal Cell Culture. Alan R Liss: New York, 1984, pp 195-205.

41 Elroy-Stein O, Fuerst TR, Moss B. Cap-independent translation of mRNA conferred by encephalomyocarditis virus $5^{\prime}$ sequence improves the performance of the vaccinia virus/bacteriophage T7 hybrid expression system. Proc Natl Acad Sci USA 1989; 86: 6126-6130.

42 Malone RW, Felgner PL, Verma IM. Cationic liposome-mediated RNA transfection. Proc Natl Acad Sci USA 1989; 86: 6077-6081.

43 Gorman CM, Moffat LF, Howard BH. Recombinant genomes which express chloramphenicol acetyltransferase in mammalian cells. Mol Cell Biol 1982; 2: 1044-1051.

44 Leonard WJ et al. Molecular cloning and expression of cDNAs for the human interleukin-2 receptor. Nature 1984; 311: 626-631.

45 Goldstein S, Fordis CM, Howard BH. Enhanced transfection efficiency and improved cell survival after electroporation of G2/M-synchronized cells and treatment with sodium butyrate. Nucleic Acids Res 1989; 17: 3959-3971.

46 Fordis CM, Howard BH. Use of the CAT reporter gene for optimization of gene transfer into eukaryotic cells. Meth Enzymol 1987; 151: 382-397. 